\title{
The characteristics of understanding of
} depression among older patients treated with
antidepressants: a comparison between older
and younger patients

This article was published in the following Dove Press journal:

Neuropsychiatric Disease and Treatment

\author{
Tetsu Tomita' \\ Shuhei Kudo ${ }^{2}$ \\ Norio Sugawara ${ }^{3}$ \\ Akira Fujii ${ }^{4}$ \\ Koji Tsuruga ${ }^{5}$ \\ Yasushi Sato' \\ Masamichi Ishioka ${ }^{6}$ \\ Kazuhiko Nakamura' \\ Norio Yasui-Furukori' \\ 'Department of Neuropsychiatry, \\ Graduate School of Medicine, \\ Hirosaki University, Hirosaki, Japan; \\ ${ }^{2}$ Department of Psychiatry, Tsugaru \\ General Hospital, Goshogawara, Japan; \\ ${ }^{3}$ Department of Clinical Epidemiology, \\ Translational Medical Center, National \\ Center of Neurology and Psychiatry, \\ Kodaira, Japan; ${ }^{4}$ Department of \\ Mental Health, Mutsu General \\ Hospital, Mutsu, Japan; ${ }^{5}$ Department \\ of Psychiatry, Aomori Prefectural \\ Tsukushigaoka Hospital, Aomori, \\ Japan; ${ }^{6}$ Department of Psychiatry, \\ Minato Hospital, Hachinohe, Japan
}

Background: To reveal characteristics of understanding of depression among older patients, we reanalyzed the data from a previous study of patients who were administered antidepressants.

Methods: A total of 424 outpatients were enrolled in this study. We used an original selfadministered questionnaire consisting of eight items: depressive symptoms, the course of depression, the cause of depression, the treatment plan, the duration of antidepressant use, how to discontinue antidepressants, the side effects of antidepressants, and psychotherapy. Each item consisted of the following two questions: "Have you received an explanation from the doctor in charge?" and "How much do you understand about your treatment?". The level of understanding was rated on a scale of 0-10 (11 anchor points). Subjects were divided into two groups: younger patients who were $<65$ years of age and older patients who were $\geq 65$ years of age.

Results: Older patients with depression showed lower levels of understanding of depression and did not receive sufficient psychoeducation from their physicians, but their understanding of depression might not be associated with their remission. In the younger group, the scores of understanding of the course of depression, the treatment plan, how to discontinue antidepressants, and psychotherapy items, and the total understanding score of remitters, were significantly higher than those of non-remitters. In contrast, there were no significant differences in the items score or total score between remitters and non-remitters in the older group.

Conclusion: Older patients showed lower levels of understanding of depression and did not appear to receive sufficient psychoeducation, but their understanding of depression might not be associated with their remission.

Keywords: depression, psychoeducation, older patients, younger patients

\section{Introduction}

The number of older patients with depression has increased in recent years, and the prevalence rate of depression has been estimated to be over $30 \%$ in this population. ${ }^{1-3}$ Older patients with depression suffer frequent relapse and poor or incomplete response to treatment ${ }^{4-6}$ because depression is related to cognitive decline, the risk of dementia and suicide, and overall mortality. ${ }^{7-9}$ Depression is an important issue for the older people, so effective treatment and public support is needed to treat them and prevent the progression of subsequent disorders.

The early stage of treating patients with depression includes several problems. The first problem is low participation rate. A study showed that only $18.1 \%$ of the
Correspondence: Tetsu Tomita Department of Neuropsychiatry, Graduate School of Medicine, Hirosaki University, 5 Zaifu-cho, Hirosaki 036-8562, Japan

Tel $+8 \mid \quad I 72395066$

Fax +8I 172395067

Email ttomita1984@yahoo.co.jp
Neuropsychiatric Disease and Treatment 2018:14 |319-1327

1319

Dovepress in

http://dx.doi.org/10.2147/NDTS158254 (c) (1) (-) 2018 Tomita et al. This work is published and licensed by Dove Medical Press Limited. The full terms of this license are available at https://www.dovepress.com/terms.php cc) hereby accept the Terms. Non-commercial uses of the work are permitted without any further permission from Dove Medical Press Limited, provided the work is properly attributed. For permission for commercial use of this work, please see paragraphs 4.2 and 5 of our Terms (https://www.dovepress.com/terms.php). 
patients with depression saw psychiatrists. ${ }^{10}$ The second problem is low continuation rate of taking medication to treat their depression after they began taking medication. A previous study reported that over half of antidepressants were stopped by the patients with depression before 6 months and that over $60 \%$ of their physicians were not consulted before discontinuation. ${ }^{11}$

Psychoeducation has become one of the standard treatments for several psychiatric disorders. The study by Brown et al showed that in the 1970 s the families of patients with schizophrenia were provided psychoeducation. ${ }^{12}$ Kemp et al showed that "compliance therapy" was effective in improving compliance and treatment efficacy in patients with schizophrenia. ${ }^{13}$ In a psychoeducation study of patients with bipolar disorder, a brief psychoeducation intervention combined with a pharmacological treatment was showed to be more effective in improving subjective quality of life of patients than a pharmacological intervention alone. ${ }^{14}$ Similarly, the effectiveness or importance of psychoeducation for patients with depression has been demonstrated by many psychoeducation studies of patients with depression.

For example, some studies have reported that antidepressant adherence might be increased by psychoeducation ${ }^{15,16}$ and that the symptoms of patients with depression were decreased by psychoeducation ${ }^{17}$ and relapse was effectively prevented. ${ }^{18,19}$ In a meta-analysis, Donker et al concluded that brief passive psychoeducation interventions could reduce patient symptoms. ${ }^{20}$ According to the study of Brown et al, providing key information about antidepressants to patients at baseline might improve adherence to antidepressants (eg, "told what to do if there were questions", "told how long to expect to take medicine", "advised of how long side effects will last", and "given advice on managing minor side effects"). ${ }^{21}$ It is important to provide these educational messages to patients with depression in clinical settings, but authors have reported that not all of the patients received enough information. ${ }^{21}$

Thus, psychoeducation is important for patients with depression, especially for older patients. Because the actual rate of administration of psychoeducation for patients with depression and the level of understanding of depression among patients with depression had not been deeply examined, we previously surveyed the understanding of depression of outpatients who had experienced or were experiencing a depressive episode and who had taken or were taking antidepressants. ${ }^{22}$ Low levels of understanding of depression by patients were revealed in our previous study, and the secondary results of the study showed the understanding of depression, which was evaluated using several questionnaires, was significantly associated with the severity of depression. ${ }^{23}$

We have already studied the characteristics of understanding of depression of patients, but there were no reports on those characteristics in older patients or on the differences in those characteristics between older and younger patients. We hypothesized that older patients had poor understanding of depression and their poor understanding was associated with poor response to treatment. In the present study, we reanalyzed the data from a previous study on the understanding of depression of older patients and compared their characteristics with younger patients.

\section{Methods \\ Participants}

We conducted this study from February to October 2013. We recruited participants who were outpatients, had experienced or were experiencing a depressive episode, and had taken or were taking antidepressants. Patients were excluded if they could not complete the questionnaire to measure their level of understanding of depression. We also excluded those patients with severe dementia, severe depression, mental retardation, and blindness. For ethical reasons, physicians excluded patients with delusions, suicidal ideation, dementia, and sub-stupor in a severe depressive state because questionnaires are invasive in these situations. In total, we enrolled 424 outpatients in the present study. The response rate was $88.3 \%$. The participants were outpatients of six hospitals in Aomori and Akita, Japan, including Hirosaki University School of Medicine and Hospital, Hirosaki Aiseikai Hospital, Kuroishi General Hospital, Mutsu General Hospital, Odate City General Hospital, and Seihoku Chuoh Hospital.

The Ethics Committee of Hirosaki University Hospital approved this study, and all of the patients or their authorized representatives provided written informed consent prior to participation.

\section{Measures}

We used a self-administered original questionnaire which consisted of the following eight items to assess the level of understanding of depression among participants: (A) depressive symptoms, (B) the course of depression, (C) the cause of depression, (D) the treatment plan, (E) the duration of antidepressant use, (F) how to discontinue antidepressants, $(\mathrm{G})$ the side effects of antidepressants, and (H) psychotherapy. The following two questions were asked for each item: "Have you received an explanation from the doctor in charge?" and "How much do you understand about your 
treatment?". After receiving explanation, each question was answered with a "yes" or "no". The participants evaluated their level of understanding by rating on an 11-point scale from 0 ("I do not understand it at all") to 10 ("I understand it perfectly"). We defined the total score of the eight items as the understanding score.

We administered the Quick Inventory of Depressive Symptomatology Japanese version (QIDS-J) to evaluate the severity of depression. The reliability and validity of this instrument have been previously established. ${ }^{24-26}$ In the present study, we defined subjects with QIDS scores $\leq 5$ as remitters. All participants were diagnosed using DSM-IV.

All participants were assessed using the Global Assessment of Functioning (GAF) and the Clinical Global Impression Severity (CGI-S) scales to evaluate their general functioning and illness severity. The GAF has a numerical scale (0-100) and is used by mental health clinicians and physicians to subjectively rate the social, occupational, and psychological functioning of adults. The CGI-S is commonly used to evaluate the severity of symptoms, treatment response, and treatment efficacy in patients with mental disorders. ${ }^{27}$ It uses a 7-point scale that requires the clinician to rate the severity of a patient's illness at the time of the assessment relative to the clinician's past experience with patients with the same diagnosis. Considering the total clinical experience, physicians rated the patients' severity of mental illness at the time of the assessment as 1 (normal, not at all ill), 2 (borderline mentally ill), 3 (mildly ill), 4 (moderately ill), 5 (markedly ill), 6 (severely ill), or 7 (extremely ill).

We collected the following data of participants from their medical records: demographics, diagnosis, age at onset, disease duration, duration of antidepressant use, number of major depressive episodes and hospitalizations, and employment status.

\section{Statistical analyses}

We compared the characteristics between remitters and nonremitters in terms of clinical and epidemiological items using chi-square tests and two-sample $t$-tests.

We divided the patients into two groups: younger group (age $<65$ ) and older group (age $\geq 65$ ). We compared the rates of remission between the younger and older groups using chi-square tests and $t$-tests.

We also analyzed the association between receiving explanations and understanding score for each item for both younger and older subjects using $t$-tests.

In addition, we compared the characteristics of receiving explanations and understanding scores between remitters and non-remitters in both the younger and older groups using chi-square tests and $t$-tests. We used $\mathrm{G}^{*}$ Power version 3.0.10 (Franz Faul, Universitat Kiel, Germany) to calculate sample size for chi-square tests and $t$-tests ( 80 and $88 \times 2$, respectively; effect size $=0.5, \alpha=0.05$ ). Each post hoc analysis achieved enough power using $\mathrm{G}^{*}$ Power.

A $p$-value of $<0.05$ was considered significant. The data were analyzed using SPSS for Windows 22 (IBM Japan, Tokyo, Japan).

\section{Results}

\section{Characteristics of the subjects and comparison between remitters and non-remitters and between younger and older groups}

Table 1 shows the characteristics of the subjects and the comparison between remitters and non-remitters and between the younger and older groups. The subjects included 134 males and 290 females, and their average, median, and mode of age were $56.1 \pm 16.9,60$, and 65 , respectively. Depression was the most frequent diagnosis among the patients.

The average age and GAF score of remitters were significantly higher than those of non-remitters. The number of major depressive episodes, CGI-S score, and QIDS-J score of remitters were significantly lower than those of non-remitters. There was no significant difference regarding the diagnostic criteria or contents of diagnosis between remitters and nonremitters. There was also no significant difference regarding receiving explanation for each item between the two groups. In contrast, the understanding scores of items B and F were significantly higher in the remitters group.

Age at onset, duration of disease, and duration of antidepressant use of the younger group were significantly lower than those of the older group. The contents of diagnosis were significantly different between the two groups. The remission rate of the older group was significantly higher than that of the younger group. The rate of receiving explanation of the younger group was significantly higher than that of the older group for all the items. The understanding scores of items A, B, C, D, G, and $\mathrm{H}$ and the total understanding score of the younger group were significantly higher than those of the older group.

\section{Comparison of the understanding scores between the groups and their association with receiving explanations}

Table 2 shows the comparison of the understanding scores between situations where the subjects received or had not 
Table I Characteristics of all subjects and the comparison between remitters and non-remitters and between younger and older groups

\begin{tabular}{|c|c|c|c|c|c|c|c|}
\hline Variables & $\begin{array}{l}\text { All subjects } \\
(n=424)\end{array}$ & $\begin{array}{l}\text { Remitters } \\
(n=\mid 22)\end{array}$ & $\begin{array}{l}\text { Non-remitters } \\
(n=269)\end{array}$ & $p$-value & $\begin{array}{l}\text { Younger } \\
(n=248)\end{array}$ & $\begin{array}{l}\text { Older } \\
(n=\mid 45)\end{array}$ & $p$-value \\
\hline Age & $56.1 \pm 16.9$ & $59.7 \pm 15.0$ & $53.2 \pm 17.3$ & $0.000 * *$ & $45.0 \pm 12.6$ & $72.7 \pm 5.5$ & $0.000 * *$ \\
\hline Sex (male:female) & $134: 290$ & $47: 75$ & $77: 192$ & 0.051 & $84: 164$ & $41: 104$ & 0.250 \\
\hline \multicolumn{8}{|l|}{ Diagnosis (n) } \\
\hline Depression & 364 & 110 & 224 & 0.123 & 199 & 137 & $0.002^{* *}$ \\
\hline Bipolar disorder & 27 & 8 & 19 & & 21 & 6 & \\
\hline Dysthymic disorder & 10 & 0 & 10 & & 10 & 0 & \\
\hline Personality disorder & 10 & 2 & 7 & & 8 & I & \\
\hline Others & 13 & 2 & 9 & & 10 & I & \\
\hline Age at onset & $47.6 \pm 16.9$ & $51.7 \pm 16.5$ & $45.1 \pm 16.9$ & $0.000 * *$ & $37.9 \pm 12.7$ & $62.7 \pm 11.3$ & $0.000^{* *}$ \\
\hline Duration of disease (years) & $8.8 \pm 9.0$ & $8.4 \pm 8.6$ & $8.5 \pm 9.0$ & 0.964 & $7.2 \pm 6.8$ & $10.7 \pm 11.2$ & $0.00 I^{* *}$ \\
\hline Duration of taking antidepressants (years) & $6.9 \pm 7.2$ & $6.8 \pm 7.3$ & $6.5 \pm 6.9$ & 0.667 & $5.4 \pm 5.5$ & $8.8 \pm 8.7$ & $0.000^{* *}$ \\
\hline Number of major depressive episodes (times) & $1.9 \pm 1.6$ & $1.6 \pm 1.4$ & $2.0 \pm 1.7$ & $0.029 *$ & $1.9 \pm 1.6$ & $1.7 \pm 1.5$ & 0.192 \\
\hline Number of hospitalizations (times) & $0.4 \pm 1.1$ & $0.4 \pm 1.1$ & $0.4 \pm 1.0$ & 0.947 & $0.4 \pm 1.1$ & $0.4 \pm 0.8$ & 0.492 \\
\hline GAF & $66.1 \pm 13.5$ & $71.4 \pm 12.2$ & $63.1 \pm 13.2$ & $0.000 * *$ & $66.7 \pm 12.9$ & $64.0 \pm 14.1$ & 0.054 \\
\hline CGI-S & $3.3 \pm 1.0$ & $2.9 \pm 0.9$ & $3.6 \pm 0.9$ & $0.000 * *$ & $3.4 \pm 0.9$ & $3.4 \pm 1.0$ & 0.899 \\
\hline QIDS-J & $9.2 \pm 5.7$ & $2.9 \pm 1.7$ & $12.1 \pm 4.4$ & $0.000 * *$ & $10.0 \pm 5.8$ & $7.9 \pm 5.3$ & $0.000^{* *}$ \\
\hline Remitters/non-remitters & $122: 269$ & & & & $68: 180$ & $56: 89$ & $0.021^{*}$ \\
\hline \multicolumn{8}{|l|}{ Receiving explanation (yes:no) } \\
\hline A & $261: 161$ & $76: 46$ & $166: 102$ & 0.947 & $180: 67$ & $64: 81$ & $0.000^{* *}$ \\
\hline B & $206: 213$ & $67: 55$ & $126: 139$ & 0.178 & $147: 99$ & $48: 95$ & $0.000^{* *}$ \\
\hline C & $213: 206$ & $62: 58$ & $137: 130$ & 0.946 & $154: 92$ & 47:96 & $0.000^{* *}$ \\
\hline $\mathrm{D}$ & 239:179 & $68: 54$ & $152: 113$ & 0.765 & $166: 80$ & $56: 87$ & $0.000^{* *}$ \\
\hline$E$ & 193:224 & $54: 68$ & $124: 14 \mid$ & 0.643 & $132: 114$ & $48: 95$ & $0.000^{* *}$ \\
\hline $\mathrm{F}$ & II9:298 & $35: 87$ & $75: 191$ & 0.920 & $84: 163$ & $28: 115$ & $0.002^{* *}$ \\
\hline G & $211: 206$ & $58: 63$ & $143: 124$ & 0.304 & $167: 80$ & $35: 108$ & $0.000^{* *}$ \\
\hline $\mathrm{H}$ & 150:265 & $42: 79$ & $101: 165$ & 0.538 & $115: 130$ & $29: 115$ & $0.000^{* *}$ \\
\hline \multicolumn{8}{|l|}{ Understanding scores } \\
\hline A & $5.2 \pm 2.8$ & $5.4 \pm 2.9$ & $5.1 \pm 2.8$ & 0.391 & $5.8 \pm 2.5$ & $4.3 \pm 3.0$ & $0.000^{* *}$ \\
\hline B & $4.6 \pm 2.9$ & $5.1 \pm 3.1$ & $4.4 \pm 2.8$ & $0.026^{*}$ & $5.0 \pm 2.8$ & $3.9 \pm 3.1$ & $0.000 * *$ \\
\hline C & $5.0 \pm 3.0$ & $5.0 \pm 3.2$ & $5.0 \pm 2.9$ & 0.917 & $5.7 \pm 2.7$ & $3.9 \pm 3.1$ & $0.000^{* *}$ \\
\hline $\mathrm{D}$ & $4.7 \pm 3.0$ & $5.0 \pm 3.1$ & $4.6 \pm 3.0$ & 0.216 & $5.3 \pm 2.8$ & $3.7 \pm 3.2$ & $0.000^{* *}$ \\
\hline $\mathrm{E}$ & $3.9 \pm 3.1$ & $4.3 \pm 3.3$ & $3.7 \pm 3.0$ & 0.073 & $4.1 \pm 3.0$ & $3.6 \pm 3.4$ & 0.076 \\
\hline $\mathrm{F}$ & $3.2 \pm 3.0$ & $3.7 \pm 3.3$ & $2.8 \pm 2.8$ & $0.009 * *$ & $3.3 \pm 2.8$ & $2.8 \pm 3.2$ & 0.092 \\
\hline G & $3.9 \pm 3.1$ & $4.4 \pm 3.3$ & $3.7 \pm 3.0$ & 0.056 & $4.6 \pm 2.9$ & $2.8 \pm 3.0$ & $0.000^{* *}$ \\
\hline $\mathrm{H}$ & $3.3 \pm 3.0$ & $3.7 \pm 3.2$ & $3.1 \pm 2.9$ & 0.050 & $3.8 \pm 2.9$ & $2.4 \pm 2.8$ & $0.000 * *$ \\
\hline Total & $33.8 \pm 20.2$ & $35.8 \pm 22.2$ & $32.7 \pm 19.1$ & 0.251 & $37.7 \pm 17.9$ & $27.2 \pm 22.0$ & $0.000^{* *}$ \\
\hline
\end{tabular}

Notes: Data shown as number, mean $\pm \mathrm{SD}$, or $p$-value. A, depressive symptoms; B, the course of depression; C, the cause of depression; $D$, the treatment plan; E, the duration of antidepressant uses; F, how to discontinue antidepressant; G, the side effects of antidepressants; H, psychotherapy. ${ }^{*} p<0.05 ;{ }^{* *} p<0.01$.

Abbreviations: GAF, Global Assessment of Functioning; CGI-S, Clinical Global Impression - Severity; QIDS-J, Quick Inventory of Depressive Symptomatology Japanese version.

received explanations for each item in the two groups. For example, the top of the table shows the comparison of the understanding scores of item A between the subjects who had received an explanation of item $\mathrm{A}$ and the subjects who had not received an explanation, for both the younger and older groups.

All of the comparisons showed significant differences between the subjects who had and had not received explanations. In all cases, the subjects who received explanations showed higher understanding scores.
Comparison between remitters and non-remitters in both younger and older groups

Table 3 shows the comparison between remitters and nonremitters in both the younger and older groups. In the younger group, the average age and age at onset of remitters were significantly higher than those of non-remitters, and the number of male remitters was high among the younger group.

Regarding receiving an explanation, no items showed significant difference between remitters and non-remitters 
Table 2 Comparison of the understanding scores between the groups and their association with receiving explanations

\begin{tabular}{|c|c|c|c|c|c|c|c|}
\hline \multirow{2}{*}{$\begin{array}{l}\text { Receiving } \\
\text { explanation }\end{array}$} & \multirow{2}{*}{$\begin{array}{l}\text { Understanding } \\
\text { scores }\end{array}$} & \multicolumn{3}{|l|}{ Younger } & \multicolumn{3}{|l|}{ Older } \\
\hline & & $\begin{array}{l}\text { Received: } \\
\text { mean } \pm \text { SD, n }\end{array}$ & $\begin{array}{l}\text { Not received: } \\
\text { mean } \pm \text { SD, n }\end{array}$ & $p$-value & $\begin{array}{l}\text { Received: } \\
\text { mean } \pm \text { SD, n }\end{array}$ & $\begin{array}{l}\text { Not received: } \\
\text { mean } \pm \text { SD, } \mathbf{n}\end{array}$ & $p$-value \\
\hline \multirow[t]{9}{*}{ Item A } & A & $6.3 \pm 2.3,177$ & $4.3 \pm 2.4,65$ & 0.000 & $6.0 \pm 2.3,62$ & $3.0 \pm 2.9,80$ & 0.000 \\
\hline & B & $5.7 \pm 2.6,179$ & $3.3 \pm 2.4,66$ & 0.000 & $5.5 \pm 2.6,64$ & $2.7 \pm 2.8,81$ & 0.000 \\
\hline & C & $6.2 \pm 2.5,179$ & $4.2 \pm 2.8,66$ & 0.000 & $5.6 \pm 2.6,63$ & $2.6 \pm 2.9,81$ & 0.000 \\
\hline & $\mathrm{D}$ & $5.9 \pm 2.5,176$ & $3.5 \pm 2.8,66$ & 0.000 & $5.5 \pm 3.1,63$ & $2.4 \pm 2.7,80$ & 0.000 \\
\hline & $E$ & $4.7 \pm 2.8,178$ & $2.5 \pm 2.7,66$ & 0.000 & $5.2 \pm 3.1,62$ & $2.3 \pm 3.0,81$ & 0.000 \\
\hline & $\mathrm{F}$ & $3.7 \pm 2.9,177$ & $2.4 \pm 2.6,67$ & 0.002 & $4.4 \pm 3.4,63$ & $1.5 \pm 2.3,80$ & 0.000 \\
\hline & G & $5.1 \pm 2.9,176$ & $3.1 \pm 2.6,66$ & 0.000 & $4.0 \pm 3.4,62$ & $1.9 \pm 2.4,80$ & 0.000 \\
\hline & $\mathrm{H}$ & $4.4 \pm 2.9,176$ & $2.2 \pm 2.4,64$ & 0.000 & $3.6 \pm 3.1,62$ & $|.4 \pm 2.1,8|$ & 0.000 \\
\hline & Total & $42.1 \pm 16.3,166$ & $25.2 \pm 16.2,60$ & 0.000 & $40.2 \pm 20.1,57$ & $17.7 \pm 18.1,78$ & 0.000 \\
\hline \multirow[t]{9}{*}{ Item B } & A & $6.4 \pm 2.3,146$ & $4.8 \pm 2.6,95$ & 0.000 & $6.1 \pm 2.3,46$ & $3.4 \pm 3.0,95$ & 0.000 \\
\hline & B & $6.1 \pm 2.4,146$ & $3.6 \pm 2.6,98$ & 0.000 & $5.8 \pm 2.4,48$ & $2.9 \pm 2.8,95$ & 0.000 \\
\hline & C & $6.5 \pm 2.3,146$ & $4.5 \pm 2.9,98$ & 0.000 & $5.7 \pm 2.3,48$ & $3.0 \pm 3.1,94$ & 0.000 \\
\hline & $\mathrm{D}$ & $6.3 \pm 2.2,144$ & $3.7 \pm 2.8,97$ & 0.000 & $5.7 \pm 2.7,47$ & $2.7 \pm 3.0,94$ & 0.000 \\
\hline & $\mathrm{E}$ & $5.0 \pm 2.8,145$ & $2.9 \pm 2.8,98$ & 0.000 & $5.8 \pm 3.0,47$ & $2.4 \pm 2.9,94$ & 0.000 \\
\hline & $\mathrm{F}$ & $3.8 \pm 2.9,145$ & $2.7 \pm 2.6,98$ & 0.004 & $4.7 \pm 3.3,48$ & $1.8 \pm 2.7,93$ & 0.000 \\
\hline & G & $5.1 \pm 2.9,143$ & $3.8 \pm 2.8,98$ & 0.000 & $4.1 \pm 3.3,46$ & $2.1 \pm 2.7,94$ & 0.001 \\
\hline & $\mathrm{H}$ & $4.6 \pm 2.9,143$ & $2.7 \pm 2.6,96$ & 0.000 & $3.8 \pm 3.1,47$ & $1.7 \pm 2.3,94$ & 0.000 \\
\hline & Total & $43.5 \pm 15.5,137$ & $28.9 \pm 17.6,88$ & 0.000 & $42.7 \pm 18.4,42$ & $19.7 \pm 19.4$ & 0.000 \\
\hline \multirow[t]{9}{*}{ Item C } & A & $6.4 \pm 2.3,152$ & $4.7 \pm 2.6,89$ & 0.000 & $6.3 \pm 2.5,45$ & $3.3 \pm 2.8,95$ & 0.000 \\
\hline & B & $5.9 \pm 2.4,153$ & $3.7 \pm 2.8,91$ & 0.000 & $5.9 \pm 2.6,47$ & $2.8 \pm 2.7,96$ & 0.000 \\
\hline & C & $6.6 \pm 2.1,153$ & $4.2 \pm 3.0,92$ & 0.000 & $5.9 \pm 2.2,47$ & $2.9 \pm 3.0,96$ & 0.000 \\
\hline & $\mathrm{D}$ & $6.1 \pm 2.2,150$ & $3.9 \pm 3.0,91$ & 0.000 & $5.8 \pm 2.8,46$ & $2.7 \pm 2.9,95$ & 0.000 \\
\hline & $E$ & $4.9 \pm 2.7,153$ & $2.8 \pm 2.9,90$ & 0.000 & $5.4 \pm 3.1,46$ & $2.6 \pm 3.1,96$ & 0.000 \\
\hline & $\mathrm{F}$ & $3.8 \pm 2.8,152$ & $2.5 \pm 2.7,91$ & 0.001 & $4.7 \pm 3.5,47$ & $1.8 \pm 2.6,95$ & 0.000 \\
\hline & G & $5.2 \pm 2.8,|5|$ & $3.5 \pm 2.9,91$ & 0.000 & $4.1 \pm 3.6,45$ & $2.1 \pm 2.5,95$ & 0.001 \\
\hline & $\mathrm{H}$ & $4.4 \pm 2.8,15 \mathrm{I}$ & $2.8 \pm 2.8,89$ & 0.000 & $3.9 \pm 3.2,46$ & $1.6 \pm 2.2,96$ & 0.000 \\
\hline & Total & $43.4 \pm|4.8,14|$ & $28.0 \pm 18.6,85$ & 0.000 & $43 . I \pm 20.2,4 I$ & $19.8 \pm 18.5,93$ & 0.000 \\
\hline \multirow[t]{9}{*}{ Item D } & A & $6.2 \pm 2.3,164$ & $4.9 \pm 2.8,78$ & 0.000 & $6.2 \pm 2.3,55$ & $3 . I \pm 2.8,86$ & 0.000 \\
\hline & B & $5.8 \pm 2.5,165$ & $3.5 \pm 2.8,80$ & 0.000 & $6.0 \pm 2.5,56$ & $2.6 \pm 2.6,87$ & 0.000 \\
\hline & $\mathrm{C}$ & $6.3 \pm 2.4,165$ & $4.5 \pm 3.0,79$ & 0.000 & $6.1 \pm 2.4,55$ & $2.5 \pm 2.8,87$ & 0.000 \\
\hline & $\mathrm{D}$ & $6.2 \pm 2.1,163$ & $3.3 \pm 2.9,78$ & 0.000 & $6.2 \pm 2.5,55$ & $2.2 \pm 2.6,86$ & 0.000 \\
\hline & $\mathrm{E}$ & $5.0 \pm 2.8,164$ & $2.4 \pm 2.7,79$ & 0.000 & $5.9 \pm 2.9,54$ & $2.1 \pm 2.7,87$ & 0.000 \\
\hline & $\mathrm{F}$ & $3.8 \pm 2.8,163$ & $2.5 \pm 2.8,80$ & 0.001 & $5.1 \pm 3.2,55$ & $1.3 \pm 2.1,86$ & 0.000 \\
\hline & G & $5.0 \pm 2.9,162$ & $3.7 \pm 2.9,79$ & 0.001 & $4.8 \pm 3.1,54$ & $1.5 \pm 2.2,86$ & 0.000 \\
\hline & $\mathrm{H}$ & $4.5 \pm 2.8,16 \mathrm{I}$ & $2.6 \pm 2.8,78$ & 0.000 & $4.2 \pm 3.1,54$ & I. $3 \pm 2.0,87$ & 0.000 \\
\hline & Total & $42.6 \pm 15.7,153$ & $27.5 \pm 18.1,73$ & 0.000 & $45.0 \pm 18.3,50$ & $16.3 \pm 16.3,84$ & 0.000 \\
\hline \multirow[t]{9}{*}{ Item E } & A & $6.3 \pm 2.4,130$ & $5.2 \pm 2.6,112$ & 0.000 & $6.1 \pm 2.4,47$ & $3.4 \pm 2.9,94$ & 0.000 \\
\hline & B & $6.0 \pm 2.5,132$ & $4.0 \pm 2.7,113$ & 0.000 & $5.8 \pm 2.5,48$ & $2.9 \pm 2.9,95$ & 0.000 \\
\hline & C & $6.4 \pm 2.4,|3|$ & $4.9 \pm 2.9,113$ & 0.000 & $6.2 \pm 2.2,48$ & $2.7 \pm 2.9,94$ & 0.000 \\
\hline & $\mathrm{D}$ & $6.4 \pm 2.1,|3|$ & $3.9 \pm 2.8,110$ & 0.000 & $5.8 \pm 2.5,47$ & $2.7 \pm 3.1,94$ & 0.000 \\
\hline & $E$ & $5.9 \pm 2.4,130$ & $2.2 \pm 2.3,113$ & 0.000 & $6.4 \pm 2.5,48$ & $2.1 \pm 2.8,93$ & 0.000 \\
\hline & $\mathrm{F}$ & $4.0 \pm 2.8,130$ & $2.6 \pm 2.7,113$ & 0.000 & $5.0 \pm 3.1,48$ & $1.6 \pm 2.6,93$ & 0.000 \\
\hline & G & $5.3 \pm 2.9,130$ & $3.8 \pm 2.8,111$ & 0.000 & $4.4 \pm 3.2,47$ & $2.0 \pm 2.6,93$ & 0.000 \\
\hline & $\mathrm{H}$ & $4.7 \pm 2.9,128$ & $2.9 \pm 2.7,111$ & 0.000 & $4.4 \pm 2.9,47$ & I. $4 \pm 2.2,94$ & 0.000 \\
\hline & Total & $44.9 \pm 16.2,121$ & $29.5 \pm 16.2,105$ & 0.000 & $43.6 \pm 17.5,45$ & $18.6 \pm 19.1,89$ & 0.000 \\
\hline \multirow[t]{9}{*}{ Item F } & A & $6.5 \pm 2.2,83$ & $5.4 \pm 2.6,159$ & 0.001 & $6.9 \pm 1.9,27$ & $3.7 \pm 3.0,114$ & 0.000 \\
\hline & B & $6.1 \pm 2.3,84$ & $4.5 \pm 2.8,16 \mid$ & 0.000 & $6.3 \pm 1.8,28$ & $3.2 \pm 3.0,115$ & 0.000 \\
\hline & C & $6.3 \pm 2.4,83$ & $5.4 \pm 2.9,162$ & 0.012 & $6.5 \pm 1.8,28$ & $3.3 \pm 3.1,114$ & 0.000 \\
\hline & $\mathrm{D}$ & $6.2 \pm 2.3,82$ & $4.8 \pm 2.9,160$ & 0.000 & $6.2 \pm 2.4,28$ & $3.1 \pm 3.1,113$ & 0.000 \\
\hline & $E$ & $5.6 \pm 2.5,84$ & $3.4 \pm 2.9,160$ & 0.000 & $6.5 \pm 1.9,28$ & $2.8 \pm 3.2,113$ & 0.000 \\
\hline & $\mathrm{F}$ & $5.8 \pm 2.2,83$ & $2.0 \pm 2.2,16 \mid$ & 0.000 & $6.1 \pm 2.2,28$ & $2.0 \pm 2.9,113$ & 0.000 \\
\hline & G & $5.6 \pm 2.8,83$ & $4.0 \pm 2.9,159$ & 0.000 & $5.5 \pm 2.5,28$ & $2.1 \pm 2.7,112$ & 0.000 \\
\hline & $\mathrm{H}$ & $4.9 \pm 2.7,83$ & $3.2 \pm 2.9,157$ & 0.000 & $4.9 \pm 2.8,28$ & $1.7 \pm 2.4,113$ & 0.000 \\
\hline & Total & $47.0 \pm 14.9,78$ & $32.6 \pm 17.4,148$ & 0.000 & $48.3 \pm 13.7,27$ & $21.5 \pm 20.1,107$ & 0.000 \\
\hline
\end{tabular}


Table 2 (Continued)

\begin{tabular}{|c|c|c|c|c|c|c|c|}
\hline \multirow{2}{*}{$\begin{array}{l}\text { Receiving } \\
\text { explanation }\end{array}$} & \multirow{2}{*}{$\begin{array}{l}\text { Understanding } \\
\text { scores }\end{array}$} & \multicolumn{3}{|l|}{ Younger } & \multicolumn{3}{|l|}{ Older } \\
\hline & & $\begin{array}{l}\text { Received: } \\
\text { mean } \pm \text { SD, } \mathbf{n}\end{array}$ & $\begin{array}{l}\text { Not received: } \\
\text { mean } \pm \text { SD, } n\end{array}$ & $p$-value & $\begin{array}{l}\text { Received: } \\
\text { mean } \pm \text { SD, n }\end{array}$ & $\begin{array}{l}\text { Not received: } \\
\text { mean } \pm \text { SD, } \mathbf{n}\end{array}$ & $p$-value \\
\hline \multirow[t]{9}{*}{ Item G } & $A$ & $6.2 \pm 2.4,165$ & $4.9 \pm 2.6,77$ & 0.000 & $7.1 \pm 2.0,35$ & $3.4 \pm 2.8,106$ & 0.000 \\
\hline & B & $5.5 \pm 2.7,166$ & $4.0 \pm 2.7,79$ & 0.000 & $6.6 \pm 2.2,35$ & $3.0 \pm 2.8,108$ & 0.000 \\
\hline & C & $6.1 \pm 2.6,166$ & $4.8 \pm 2.8,79$ & 0.000 & $6.8 \pm 2.0,34$ & $3.0 \pm 2.9,108$ & 0.000 \\
\hline & D & $5.7 \pm 2.6,164$ & $4.3 \pm 2.8,78$ & 0.000 & $6.9 \pm 2.3,35$ & $2.8 \pm 2.8,106$ & 0.000 \\
\hline & $\mathrm{E}$ & $4.6 \pm 3.0,165$ & $3.1 \pm 2.7,79$ & 0.000 & $6.8 \pm 2.4,34$ & $2.6 \pm 3.0,107$ & 0.000 \\
\hline & $\mathrm{F}$ & $3.8 \pm 2.9,164$ & $2.4 \pm 2.6,80$ & 0.000 & $5.9 \pm 2.9,34$ & $1.8 \pm 2.6,107$ & 0.000 \\
\hline & G & $5.7 \pm 2.5,164$ & $2.3 \pm 2.5,78$ & 0.000 & $6.0 \pm 2.6,33$ & $1.8 \pm 2.4,107$ & 0.000 \\
\hline & $\mathrm{H}$ & $4.4 \pm 2.9,163$ & $2.6 \pm 2.6,77$ & 0.000 & $4.9 \pm 2.9,33$ & $1.6 \pm 2.3,108$ & 0.000 \\
\hline & Total & $41.9 \pm 16.7,156$ & $28.5 \pm 17.1,70$ & 0.000 & $51.3 \pm 14.8,32$ & $19.6 \pm 18.1,102$ & 0.000 \\
\hline \multirow[t]{9}{*}{ Item $\mathrm{H}$} & A & $6.2 \pm 2.3,114$ & $5.4 \pm 2.7,126$ & 0.010 & $6.4 \pm 2.4,29$ & $3.8 \pm 3.0,113$ & 0.000 \\
\hline & B & $5.9 \pm 2.5,114$ & $4.3 \pm 2.9,129$ & 0.000 & $6.1 \pm 2.6,296$ & $3.3 \pm 2.9,115$ & 0.000 \\
\hline & C & $6.3 \pm 2.6,114$ & $5.2 \pm 2.8,129$ & 0.001 & $6.3 \pm 2.4,29$ & $3.3 \pm 3.1,114$ & 0.000 \\
\hline & D & $6.0 \pm 2.4,113$ & $4.6 \pm 2.9,127$ & 0.000 & $6.1 \pm 2.7,28$ & $3.2 \pm 3.1,114$ & 0.000 \\
\hline & $E$ & $5.1 \pm 3.0,115$ & $3.3 \pm 2.7,127$ & 0.000 & $6.2 \pm 2.6,29$ & $2.9 \pm 3.2,113$ & 0.000 \\
\hline & $\mathrm{F}$ & $4.1 \pm 2.9,114$ & $2.6 \pm 2.6,128$ & 0.000 & $5.3 \pm 2.8,29$ & $2.1 \pm 3.0,113$ & 0.000 \\
\hline & G & $5.4 \pm 2.8,114$ & $3.8 \pm 2.9,126$ & 0.000 & $5.0 \pm 2.8,28$ & $2.3 \pm 2.8,113$ & 0.000 \\
\hline & $\mathrm{H}$ & $5.5 \pm 2.4,114$ & $2.3 \pm 2.6,125$ & 0.000 & $5.4 \pm 2.4,287$ & $1.7 \pm 2.4,114$ & 0.000 \\
\hline & Total & $44.5 \pm 16.3,109$ & $31.5 \pm 17.1,116$ & 0.000 & $47.1 \pm 17.4,27$ & $22.2 \pm 20.1,108$ & 0.000 \\
\hline
\end{tabular}

Notes: A, depressive symptoms; B, the course of depression; C, the cause of depression; D, the treatment plan; E, the duration of antidepressant uses; F, how to discontinue antidepressant; $\mathrm{G}$, the side effects of antidepressants; $\mathrm{H}$, psychotherapy.

in either the younger or the older group. The understanding scores of items B, D, F, and $\mathrm{H}$ and the total understanding score were significantly higher in the younger group compared to the older group. In contrast, neither item score nor total score showed a significant difference between remitters and non-remitters in the older group.

\section{Discussion}

In the present study, we reanalyzed the data from a previous study to investigate the understanding of depression of older patients versus younger patients and demonstrated the effect of receiving psychoeducation. According to our results, older patients with depression showed lower levels of understanding of depression and did not receive adequate psychoeducation from their physicians in the usual treatment course; however, their understanding of depression might not be associated with their remission. Older people sometimes have problems with cognitive functioning, so some patients may not remember the details of psychoeducation provided by their physician, even though they had received the psychoeducation. This is the first report to survey the understanding of depression among older patients and to study the association between the understanding of depression and responsiveness to the treatment of depression.

Table 2 shows that the patients who had received psychoeducation showed higher understanding scores of depression than those who had not received psychoeducation in both the younger and older groups. However, as shown in Table 3, receiving psychoeducation might not be associated with the understanding of depression in older patients; receiving psychoeducation (by patients) or giving psychoeducation (by physicians) did not appear to be important for the treatment of patients with depression, whereas some items or the total understanding scores of psychoeducation were associated with remission in younger patients. However, the results of the present study do not allow us to draw conclusions about whether psychoeducation for older patients had any positive effects or whether there was a need to administer psychoeducation in the first place. Psychoeducation may not directly influence remission in older patients with depression, but it may indirectly influence other factors associated with positive responses to treatment or remission. For example, receiving psychoeducation from a physician might help the older patients realize that the doctor cares for them and thus lead to a good relationship between the doctor and the patients, which increases the likelihood that the treatment is continued. In addition, psychoeducation might be beneficial for the family members who accompany older patients as it helps them understand their loved ones' depression and for the patients themselves, and to promote good treatment. In general, the level of understanding of depression may not be very important in older patients. 
Table 3 Comparison between remitters and non-remitters in both the younger and older groups

\begin{tabular}{|c|c|c|c|c|c|c|}
\hline & \multicolumn{3}{|l|}{ Younger } & \multicolumn{3}{|l|}{ Older } \\
\hline & Remitters & Non-remitters & $p$-value & Remitters & Non-remitters & $p$-value \\
\hline Age & $48.4 \pm 11.7$ & $43.8 \pm 12.7$ & $0.009 * *$ & $73.2 \pm 5.6$ & $72.3 \pm 5.5$ & 0.358 \\
\hline Sex (male:female) & $30: 38$ & $54: 126$ & $0.036 *$ & $18: 38$ & $23: 66$ & 0.412 \\
\hline \multicolumn{7}{|l|}{ Diagnosis (n) } \\
\hline Depression & 61 & 138 & 0.151 & 51 & 86 & 0.229 \\
\hline Bipolar disorder & 4 & 17 & & 4 & 2 & \\
\hline Dysthymic disorder & 0 & 10 & & 0 & 0 & \\
\hline Personality disorder & I & 7 & & I & 0 & \\
\hline Others & 2 & 8 & & 0 & I & \\
\hline Age at onset & $40.7 \pm 13.0$ & $36.8 \pm 12.4$ & $0.033^{*}$ & $64.0 \pm 11.1$ & $61.9 \pm 11.4$ & 0.263 \\
\hline Duration of disease (years) & $7.5 \pm 7.5$ & $7.1 \pm 6.5$ & 0.681 & $9.7 \pm 9.5$ & $11.4 \pm 12.2$ & 0.365 \\
\hline Duration of taking antidepressants (years) & $5.3 \pm 5.6$ & $5.4 \pm 5.5$ & 0.890 & $8.7 \pm 8.5$ & $8.8 \pm 8.8$ & 0.994 \\
\hline Number of major depressive episodes (times) & $1.6 \pm 1.4$ & $2.0 \pm 1.7$ & 0.063 & $1.5 \pm 1.4$ & $1.8 \pm 1.6$ & 0.299 \\
\hline Number of hospitalizations (times) & $0.5 \pm 1.3$ & $0.4 \pm 1.0$ & 0.349 & $0.3 \pm 0.6$ & $0.4 \pm 0.9$ & 0.137 \\
\hline GAF & $73.6 \pm 10.5$ & $64.1 \pm 12.8$ & $0.000 * *$ & $68.8 \pm 13.4$ & $61.0 \pm 13.8$ & $0.00 I^{* *}$ \\
\hline CGI-S & $2.8 \pm 0.7$ & $3.6 \pm 0.9$ & $0.000 * *$ & $3.1 \pm 1.0$ & $3.6 \pm 1.0$ & $0.006 * *$ \\
\hline QIDS-J & $2.9 \pm 1.8$ & $12.6 \pm 4.4$ & $0.000 * *$ & $2.8 \pm 1.7$ & II.I \pm 4.3 & $0.000 * *$ \\
\hline \multicolumn{7}{|l|}{ Receiving explanation (yes:no) } \\
\hline A & $51: 17$ & $129: 50$ & 0.643 & $27: 29$ & $37: 52$ & 0.433 \\
\hline B & $46: 22$ & 101:77 & 0.119 & $23: 33$ & $25: 62$ & 0.127 \\
\hline C & $43: 24$ & $111: 68$ & 0.754 & $21: 34$ & $26: 62$ & 0.285 \\
\hline$D$ & $45: 23$ & $121: 57$ & 0.787 & $25: 31$ & $31: 56$ & $0.28 \mathrm{I}$ \\
\hline$E$ & $39: 29$ & $93: 85$ & 0.473 & 17:39 & $31: 56$ & 0.514 \\
\hline $\mathrm{F}$ & $26: 42$ & $58: 121$ & 0.387 & II:45 & $17: 70$ & 0.988 \\
\hline G & $44: 24$ & $123: 56$ & 0.548 & $15: 40$ & $20: 68$ & 0.539 \\
\hline $\mathrm{H}$ & $33: 34$ & $82: 96$ & 0.656 & $10: 46$ & 19:69 & 0.586 \\
\hline \multicolumn{7}{|l|}{ Understanding scores } \\
\hline A & $6.2 \pm 2.3$ & $5.6 \pm 2.6$ & 0.077 & $4.5 \pm 3.2$ & $4.2 \pm 2.9$ & 0.545 \\
\hline B & $5.9 \pm 2.6$ & $4.7 \pm 2.8$ & $0.002 *$ & $4.2 \pm 3.3$ & $3.7 \pm 2.9$ & $0.38 \mathrm{I}$ \\
\hline C & $6.1 \pm 2.7$ & $5.5 \pm 2.7$ & 0.185 & $3.8 \pm 3.3$ & $4.0 \pm 3.1$ & 0.738 \\
\hline $\mathrm{D}$ & $5.8 \pm 2.6$ & $5.0 \pm 2.8$ & $0.044^{*}$ & $4.1 \pm 3.5$ & $3.6 \pm 3.1$ & $0.40 \mathrm{I}$ \\
\hline$E$ & $4.7 \pm 2.9$ & $3.9 \pm 3.0$ & 0.057 & $3.9 \pm 3.7$ & $3.3 \pm 3.2$ & 0.302 \\
\hline $\mathrm{F}$ & $4.1 \pm 3.1$ & $3.0 \pm 2.7$ & $0.006 * *$ & $3.3 \pm 3.5$ & $2.4 \pm 2.9$ & 0.119 \\
\hline G & $5.1 \pm 3.0$ & $4.3 \pm 2.9$ & 0.066 & $3.4 \pm 3.4$ & $2.4 \pm 2.7$ & 0.062 \\
\hline $\mathrm{H}$ & $4.7 \pm 3.0$ & $3.5 \pm 2.9$ & $0.008 * *$ & $2.7 \pm 3.1$ & $2.2 \pm 2.6$ & 0.308 \\
\hline Total & $42.0 \pm 18.6$ & $36.0 \pm 17.4$ & $0.025^{*}$ & $28.7 \pm 24.1$ & $26.2 \pm 20.6$ & 0.511 \\
\hline
\end{tabular}

Notes: Data shown as number, mean \pm SD, or p-value. A, depressive symptoms; B, the course of depression; C, the cause of depression; $D$, the treatment plan; $E$, the duration of antidepressant uses; F, how to discontinue antidepressant; $G$, the side effects of antidepressants; $\mathrm{H}$, psychotherapy. $* p<0.05 ; * * p<0.0 \mathrm{I}$.

Abbreviations: GAF, Global Assessment of Functioning; CGI-S, Clinical Global Impression - Severity; QIDS-J, Quick Inventory of Depressive Symptomatology Japanese version.

In the younger group, remitters showed higher scores of understanding for items $\mathrm{B}, \mathrm{D}, \mathrm{F}$, and $\mathrm{H}$ and higher total scores than non-remitters. In the present study, we did not examine why there was such a difference. On the other hand, there was no difference between remitters and non-remitters in the rate of receiving psychoeducation for items B, D, F, and $\mathrm{H}$. These contents include prospective meanings and additional choices in drug treatment, so the patients who showed remission appeared to understand these themes more than the non-remitters.

The understanding of some items of psychoeducation might be associated with remission in younger patients; on the other hand, the understanding of psychoeducation was not associated with remission in older patients. In a future study, we should study the understanding of additional items regarding depression that we did not cover in the present study and whether they are associated with remission or response in older patients with depression. Older patients with depression are more likely to have dementia or cognitive impairment and somatic disorders related to overall mortality, ${ }^{7-9}$ so in future research it might be beneficial to study items regarding cognitive impairment and somatic symptoms to evaluate the difference between younger patients and older patients or between remitters and non-remitters in both the age groups. 
The present study has some limitations. First, we only performed subjective evaluations on receiving psychoeducation and understanding the items of psychoeducation, and "psychoeducation" in the present study includes only explanation or information. The results may not reflect the actual situation of giving, receiving, and understanding psychoeducation in clinical settings. The scoring of understanding of the items of psychoeducation might need more validation. Therefore, we should use objective evaluation scales in future studies. Psychoeducation is complex and is a kind of comprehensive intervention, so other types of questionnaires might be better. Second, the difference in the background of the two groups might cause bias in the present study. Older group included more remitters and showed lower QIDS score, and we excluded patients who were clearly diagnosed with dementia but did not evaluate their cognitive functioning. The difference in severity might have influenced the difference in the characteristics of associations between understandings and response. Some of the subjects might have had cognitive impairment, especially among the older patients. It is possible that they did not accurately recognize their understanding of psychoeducation or whether they had received the psychoeducation. Third, our study was retrospective. We did not discuss when psychoeducation was provided or received or how patients received their psychoeducation, for example, from physicians, lectures, books, TV programs, or Internet media. The present study does not provide an answer to whether understanding of the items of psychoeducation either causes or results from remission. In the future, we should plan a prospective study to understand the effect of psychoeducation and the causal relationship between psychoeducation and remission.

\section{Conclusion}

We showed the differences in receiving and understanding of psychoeducation on depression between the older and younger patients. Older patients with depression showed lower levels of understanding of depression and did not appear to receive sufficient psychoeducation from their physicians in the usual course of treatment, but their understanding of depression might not be associated with remission. In the future, more effective and beneficial psychoeducation for older patients with depression is recommended.

\section{Acknowledgments}

The authors would like to thank all their coworkers of this study for their skillful assistance in collecting and managing the data. This study was funded by a Grant-in-Aid for Scientific Research (KAKENHI) from the Japan Society for the Promotion of Research (JSPS; \#20333734 and 15H04754), Mitsubishi Pharma Research Foundation, Asteras Schizophrenia Research Foundation, and a grant from the Hirosaki Research Institute for Neurosciences. The funders had no role in study design, data collection and analysis, decision to publish, or preparation of the manuscript.

\section{Disclosure}

Norio Yasui-Furukori has received grant and/or research support or honoraria from and has been a lecturer for Asteras, Dainippon, Eli Lilly, GSK, Janssen-Pharma, Meiji, Mochida, MSD, Otsuka, Pfizer, Takeda, and Yoshitomi. The authors report no other conflicts of interest in this work.

\section{References}

1. Alvarado BE, Zunzunegui MV, Beland F, et al. Social and gender inequalities in depressive symptoms among urban older adults of Latin America and the Caribbean. J Gerontol B Psychol Sci Soc Sci. 2007;62(4):S226-S236.

2. Castro-Costa E, Dewey M, Stewart R, et al. Prevalence of depressive symptoms and syndromes in later life in ten European countries: the SHARE study. Br J Psychiatry. 2007;191:393-401.

3. Guerra M, Prina AM, Ferri CP, et al. A comparative cross-cultural study of the prevalence of late life depression in low and middle income countries. J Affect Disord. 2016;190:362-368.

4. Mitchell AJ, Subramaniam H. Prognosis of depression in old age compared to middle age: a systematic review of comparative studies. Am J Psychiatry. 2005;162(9):1588-1601.

5. Tedeschini E, Levkovitz Y, Iovieno N, et al. Efficacy of antidepressants for late-life depression: a meta-analysis and meta-regression of placebo-controlled randomized trials. J Clin Psychiatry. 2011;72(12): $1660-1668$.

6. Beekman AT, Penninx BW, Deeg DJ, et al. The impact of depression on the well-being, disability and use of services in older adults: a longitudinal perspective. Acta Psychiatr Scand. 2002;105(1):20-27.

7. Blazer DG. Depression in late life: review and commentary. J Gerontol A Biol Sci Med Sci. 2003;58(3):249-265.

8. Brailean A, Comijs HC, Aartsen MJ, et al. Late-life depression symptom dimensions and cognitive functioning in the Longitudinal Aging Study Amsterdam (LASA). J Affect Disord. 2016;201:171-178.

9. Mirza SS, Wolters FJ, Swanson SA, et al. 10-Year trajectories of depressive symptoms and risk of dementia: a population-based study. Lancet Psychiatry. 2016;3(7):628-635.

10. Kawakami N, Takeshima T, Ono Y, et al. Twelve-month prevalence, severity, and treatment of common mental disorders in communities in Japan: preliminary finding from the World Mental Health Japan Survey 2002-2003. Psychiatry Clin Neurosci. 2005;59(4):441-452.

11. Sawada N, Uchida H, Suzuki T, et al. Persistence and compliance to antidepressant treatment in patients with depression: a chart review. BMC Psychiatry. 2009;9:38.

12. Brown GW, Birley JL, Wing JK. Influence of family life on the course of schizophrenic disorders: a replication. Br J Psychiatry. 1972;121(562): 241-258.

13. Kemp R, Hayward P, Applewhaite G, et al. Compliance therapy in psychotic patients: randomised controlled trial. $B M J .1996 ; 312(7027)$ : 345-349.

14. Cardoso Tde A, Farias Cde A, Mondin TC, et al. Brief psychoeducation for bipolar disorder: impact on quality of life in young adults in a 6-month follow-up of a randomized controlled trial. Psychiatry Res. 2014;220(3):896-902. 
15. Wells KB, Sherbourne C, Schoenbaum M, et al. Impact of disseminating quality improvement programs for depression in managed primary care: a randomized controlled trial. JAMA. 2000;283(2):212-220.

16. Rost K, Nutting $\mathrm{P}$, Smith J, et al. Improving depression outcomes in community primary care practice: a randomized trial of the quEST intervention. Quality Enhancement by Strategic Teaming. J Gen Intern Med. 2001;16(3):143-149.

17. Morokuma I, Shimodera S, Fujita H, et al. Psychoeducation for major depressive disorders: a randomised controlled trial. Psychiatry Res. 2013; 210(1):134-139.

18. Shimazu K, Shimodera S, Mino Y, et al. Family psychoeducation for major depression: randomised controlled trial. Br J Psychiatry. 2011; 198(5):385-390.

19. Kronmuller KT, Victor D, Schenkenbach C, et al. Knowledge about affective disorders and outcome of depression. J Affect Disord. 2007; 104(1-3):155-160.

20. Donker T, Griffiths KM, Cuijpers P, et al. Psychoeducation for depression, anxiety and psychological distress: a meta-analysis. BMC Med. 2009;7:79

21. Brown C, Battista DR, Sereika SM, et al. How can you improve antidepressant adherence? J Fam Pract. 2007;56(5):356-363.

22. Kudo S, Tomita T, Sugawara N, et al. The low level of understanding of depression among patients treated with antidepressants: a survey of 424 outpatients in Japan. Neuropsychiatr Dis Treat. 2015;11:2811-2816.
23. Tomita T, Yasui-Furukori N, Kudo S, et al. The association between the severity and level of understanding of depression among patients treated with antidepressants: a survey of 424 outpatients in Japan. Clin Neuropharmacol. 2018;41(1):1-5.

24. Rush AJ, Trivedi MH, Ibrahim HM, et al. The 16-Item Quick Inventory of Depressive Symptomatology (QIDS), clinician rating (QIDS-C), and self-report (QIDS-SR): a psychometric evaluation in patients with chronic major depression. Biol Psychiatry. 2003;54(5):573-583.

25. Trivedi MH, Rush AJ, Ibrahim HM, et al. The Inventory of Depressive Symptomatology, Clinician Rating (IDS-C) and Self-Report (IDS-SR), and the Quick Inventory of Depressive Symptomatology, Clinician Rating (QIDS-C) and Self-Report (QIDS-SR) in public sector patients with mood disorders: a psychometric evaluation. Psychol Med. 2004;34(1):73-82.

26. Fujisawa D, Nakagawa A, Tajima M, et al. [Reliability and validity of Quick Inventory of Depressive Symptomatology Japanese version]. Seishin Shinkeigaku Zasshi. 2008;(Supplement):S-324. Japanese [with English abstract].

27. Guy W. Clinical Global Impression Scale (CGI). ECDEU Assessment Manual for Psychopharmacology. Rockville, MD: US Department of Health, Education, and Welfare, Public Health Service, Alcohol, Drug Abuse, and Mental Health Administration, National Institute of Mental Health, Psychopharmacology Research Branch, Division of Extramural Research Programs; 1976:217-222.
Neuropsychiatric Disease and Treatment

\section{Publish your work in this journal}

Neuropsychiatric Disease and Treatment is an international, peerreviewed journal of clinical therapeutics and pharmacology focusing on concise rapid reporting of clinical or pre-clinical studies on a range of neuropsychiatric and neurological disorders. This journal is indexed on PubMed Central, the 'PsycINFO' database and CAS,

\section{Dovepress}

and is the official journal of The International Neuropsychiatric Association (INA). The manuscript management system is completely online and includes a very quick and fair peer-review system, which is all easy to use. Visit http://www.dovepress.com/testimonials.php to read real quotes from published authors. 